\title{
Detection of retinal changes in Parkinson's disease with spectral-domain optical coherence tomography
}

This article was published in the following Dove Press journal:

Clinical Ophthalmology

3 December 2010

Number of times this article has been viewed

\author{
Grant D Aaker' \\ Jane S Myung' \\ Joshua R Ehrlich' \\ Mujtaba Mohammed ${ }^{2}$ \\ Claire Henchcliffe ${ }^{2}$ \\ Szilárd Kiss' \\ 'Department of Ophthalmology, \\ ${ }^{2}$ Department of Neurology, Cornell \\ Medical College, New York, USA
}

Purpose: This pilot study investigated whether high-resolution spectral-domain optical coherence tomography (SD-OCT) could detect differences in inner retinal layer (IRL), peripapillary retinal nerve fiber layer (RNFL), and macular thickness between patients with Parkinson's disease (PD) and controls.

Methods: Both eyes of patients with PD and age-matched controls were imaged with the Heidelberg Spectralis ${ }^{\circledR}$ HRA + OCT. RNFL, IRL, and macular thickness were measured for each eye using Heidelberg software. These measurements were compared with validated, published normal values for macular and RNFL thickness, and compared with matched controls for IRL thickness.

Results: Eighteen eyes from nine subjects with PD and 19 eyes of 16 control subjects were evaluated using SD-OCT. The average age of PD patients was 64 years with a range of $52-75$ years. The average age of controls was 67 years with a range of 50-81 years. No significant reduction in IRL thickness was detected between PD patients and age-matched controls at 13 points along a $6 \mathrm{~mm}$ horizontal section through the fovea. No significant difference in RNFL thickness was detected between PD patients and published normal values. Overall average RNFL thickness was $97 \mu \mathrm{m}$ for PD patients, which exactly matched the normative database value. However, significant differences in macular thickness were detected in three of nine subfields between PD subjects and published normal values. In PD subjects, the outer superior subfield was $2.8 \%$ thinner $(P=0.026)$, while the outer nasal and inner inferior subfields were $2.8 \%$ $(P=0.016)$ and $2.7 \%(P=0.001)$ thicker compared to published normal values.

Conclusion: In this pilot study, significant differences in macular thickness were detected in three of nine subfields by SD-OCT. However, SD-OCT did not detect significant reductions in peripapillary RNFL and IRL thickness between PD patients and controls. This suggests that macular thickness measurements by SD-OCT may potentially be used as an objective, noninvasive, and easily quantifiable in vivo biomarker in PD. Larger, longitudinal studies are needed to explore these relationships further.

Keywords: Parkinson's disease, spectral-domain optical coherence tomography, nerve fiber layer thickness, macular thickness, inner retinal layer thickness

\section{Introduction}

Parkinson's disease (PD), the second most common neurodegenerative disease, is a progressive disorder with selective dopaminergic neuronal loss, mainly in the substantia nigra. It was first described by James Parkinson in $1817 .{ }^{1}$ Visual symptoms are common in PD, and include reduced spatial contrast sensitivity, motion perception abnormalities, color deficiency, and visual hallucinations. Dopaminergic neuronal cells have been identified in the inner nuclear layer and inner plexiform layers of the human 
retina. ${ }^{2}$ Histopathologic studies on postmortem Parkinson's patients never treated with levodopa showed significantly lower retinal dopamine concentrations than controls or individuals with PD whose death occurred less than 15 hours after their last dose. ${ }^{3}$ The role of retinal dopaminergic neuronal cells in visual function has been well studied in both human and primate models, and these cells appear to modulate the receptive fields of retinal ganglion cells to provide spatial contrast sensitivity and color vision. ${ }^{4-6}$

Thinning of the retinal nerve fiber layer (RNFL), the inner retinal layer (IRL), and macular thickness have been documented in several small studies, and it has been proposed that this may correlate with loss of these dopaminergic cells and progression of functional visual abnormalities in PD patients (Figure 1). ${ }^{7-10}$

Optical coherence tomography (OCT) is a noninvasive retinal imaging modality that can rapidly provide highresolution, cross-sectional images of the retina. It is the best modality for measuring retinal thickness and volume, and can precisely quantify changes in the retina through comparative imaging.

The primary goal of this pilot project was to investigate RNFL thickness, macular thickness, and IRL thickness in patients with PD using high-resolution spectral domain OCT (SD-OCT) and to compare them with unaffected control subjects to determine if reductions in the thickness of these structures could be detected in PD patients.

\section{Methods}

This study was approved by the institutional review board at Weill Cornell Medical College and New York Presbyterian Hospital, and adhered to the tenets of the Declaration of Helsinki. Consecutive patients diagnosed with PD who were referred to our clinic for ophthalmic evaluation, as well as age-matched controls, were included. All patients and

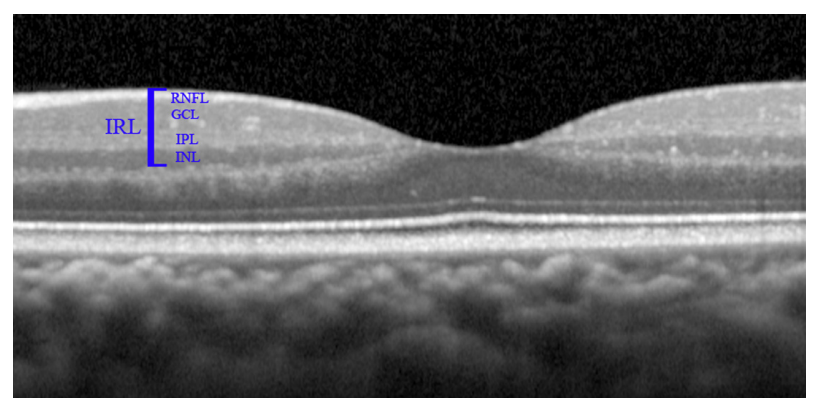

Figure I OCT image of the retina of the left eye. The layers of the inner retinal layer (IRL) are shown: retinal nerve fiber layer (RNFL), ganglion cell layer (GCL), inner plexiform layer (IPL), and inner nuclear layer (INL). Dopaminergic cells have been isolated primarily to the INL of the primate retina. controls underwent a dilated fundus examination. Patients with a history of glaucoma, optic neuropathy, age-related macular degeneration, diabetic retinopathy, or other significant retinal or optic nerve diseases were excluded.

Optic nerve and macular imaging was performed in the Department of Ophthalmology at Weill Cornell Medical College. Both eyes of each patient with PD and controls were imaged with the high-resolution, SD-OCT Heidelberg Spectralis ${ }^{\circledR}$ HRA + OCT (Heidelberg Engineering, Heidelberg, Germany) by a trained operator. Scanning protocols included a circular $3.4 \mathrm{~mm}$ scan centered on the optic nerve head, a volumetric scan of the macula centered on the fovea (73 horizontal B-scans covering a superior-to-inferior distance of $4.6 \mathrm{~mm}$ ), and a section scan through the fovea. Poor quality images were excluded from final analysis.

RNFL, IRL, and macular thickness were measured for each eye using the Heidelberg Eye Explorer software (Version 1.6.2.0). Each scan was individually reviewed and segmentation lines were adjusted to ensure accuracy in both RNFL and macular thickness measurements. RNFL measurements were recorded by sector, ie, temporal, superotemporal, superonasal, nasal, inferonasal, inferotemporal, and average (Figure 2), and compared with the Heidelberg normative database. Macular thickness was reported in a modified Early Treatment of Diabetic Retinopathy Study macular map with the central subfield $1.00 \mathrm{~mm}$ in diameter, and the inner and outer subfields having diameters of $2.22 \mathrm{~mm}$ and $3.45 \mathrm{~mm}$, respectively (Figure 3), and compared with published normals. ${ }^{11}$ IRL thickness measurements were determined for PD subjects, as well as age-matched controls, by manually segmenting the IRL (because no normal values for IRL have been published). This was accomplished using Heidelberg software by setting the inner limit of the IRL at the internal limiting membrane, and the outer limit at the outer border of the inner nuclear layer (Figure 1) and measuring the vertical thickness at 13 points at $500 \mu \mathrm{m}$ intervals along a $6 \mathrm{~mm}$ horizontal section through the fovea (Figure 4).

Mann-Whitney $U$ tests were performed to detect differences between IRL thickness in PD patients versus controls, and macular thickness versus published normals. A onesample $t$-test was used to detect differences in RNFL thickness between PD patients and normal values from the Heidelberg database. Statistical analyses were performed using Stata/ IC 11.0 (StataCorp, College Station, TX).

\section{Results}

OCT imaging of the optic nerve and macula was performed on 18 eyes of nine patients with PD and 19 eyes of 16 controls 

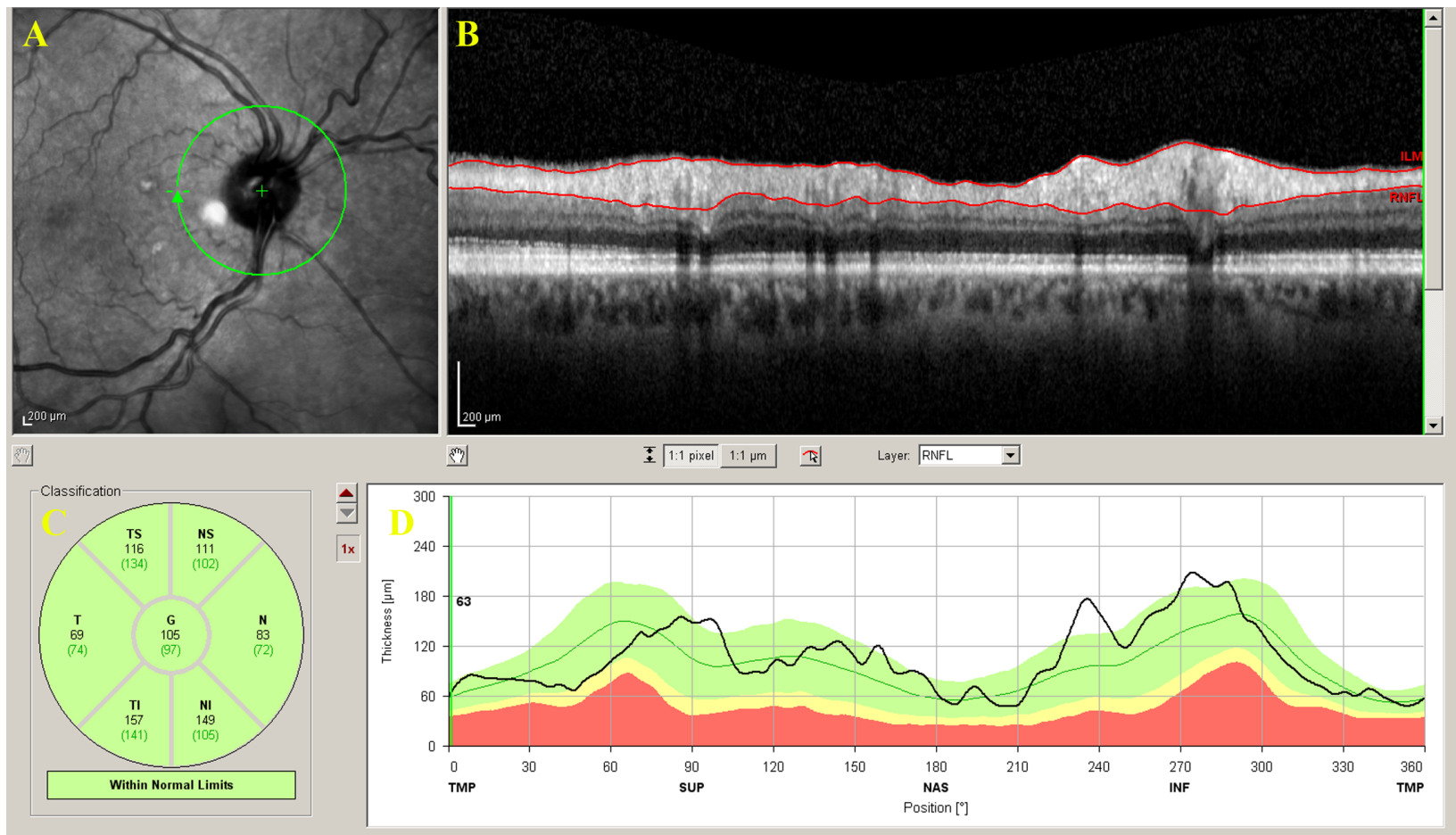

\section{8/20/2009, OD}

Figure 2 Screen grab from the Heidelberg Eye Explorer software automated analysis of the RNFL thickness. A) Infrared image showing the location of the circular scan (green circle) centered around the optic nerve (green cross) of the right eye. B) Circular optical coherence tomography image of the retina showing the RNFL segmented (red lines). C) RNFL thickness measurements in seven sectors as measured by the software. D) Plot of patient's RNFL thickness (black line) plotted against normative values (colored lines).

Abbreviation: RNFL, retinal nerve fiber layer.
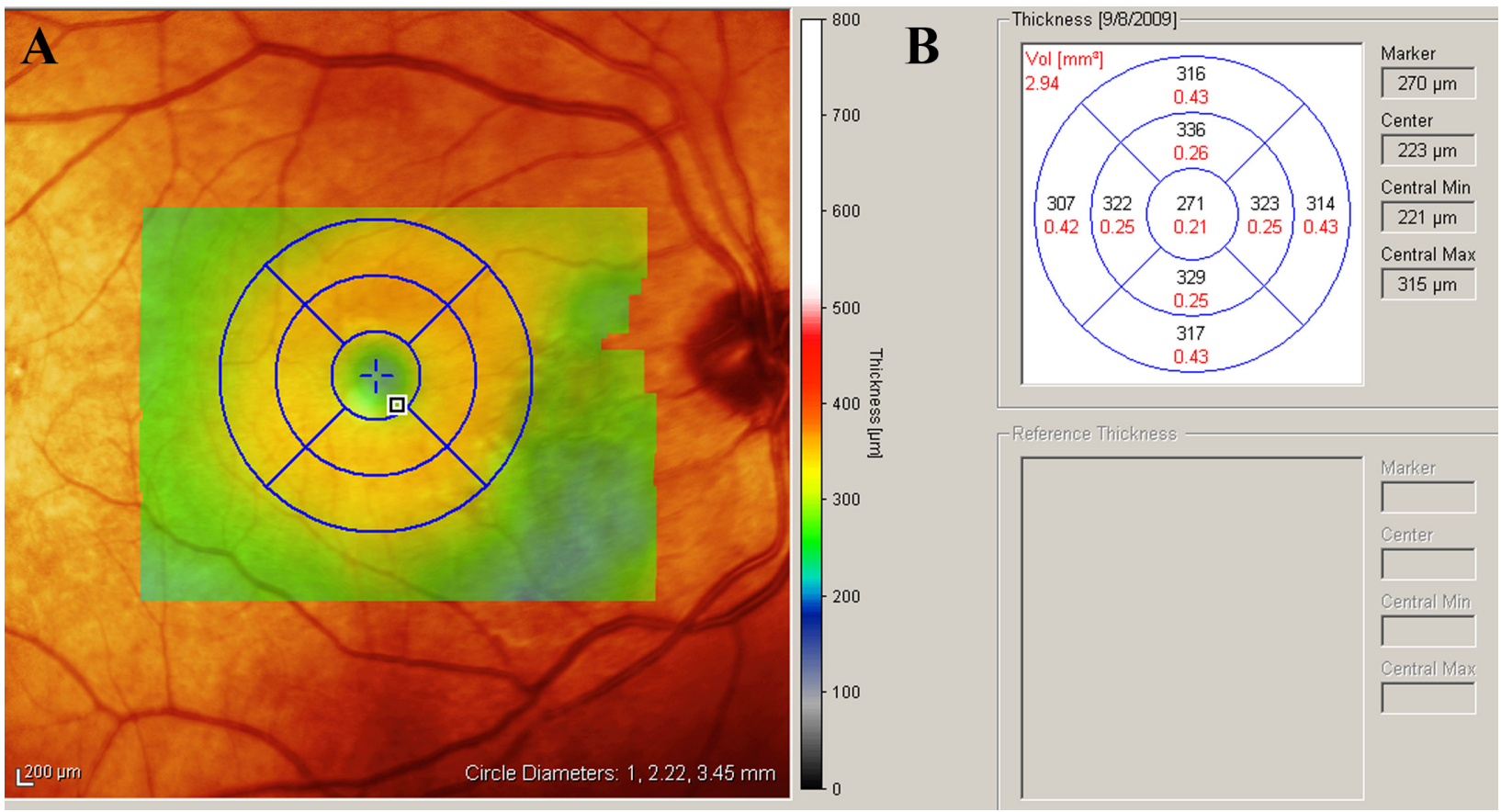

Figure 3 Screen grab from the Heidelberg Eye Explorer software analysis of macular thickness. Infrared image (A) of the posterior pole of the right eye of a Parkinson's disease patient with superimposed thickness map (multicolored box) and grid corresponding to the nine subfields measured in (B). 


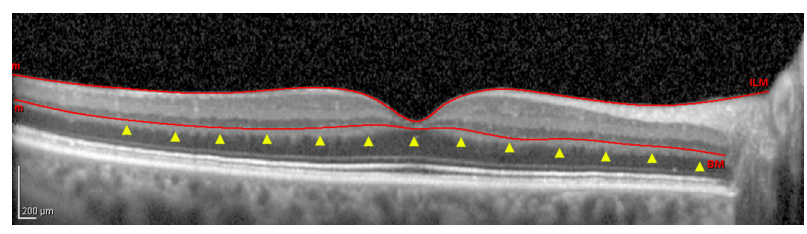

Figure 4 High-resolution spectral-domain optical coherence tomography image of a section through the fovea of the right eye of a control patient showing segmentation of the inner retinal layer (red lines). Yellow arrowheads correspond to the 13 points at which the IRL was measured in Parkinson's disease patients and age-matched controls in the study. These points were equally spaced at $500 \mu \mathrm{m}$ intervals along a $6 \mathrm{~mm}$ horizontal line centered on the fovea.

(Table 1). All images were of acceptable quality for inclusion in the study. The average age of PD patients was 64 years with a range of 52-75 years. The average age of controls was 67 years with a range of 50-81 years. Of PD patients included in the study, $44 \%$ were female, while $38 \%$ of controls were female. Severity ratings for PD patients in the study were in the range 1-3 using the modified Hoehn and Yahr scale, with two patients having Stage 3 disease, four patients having Stage 2 disease, and three patients having Stage 1 disease. $^{12}$

No significant differences were found in IRL thickness at any of the 13 points between PD patients and controls in our study (Table 2). Overall, average IRL thickness in PD patients was actually greater at all but two points measured along the $6 \mathrm{~mm}$ section through the fovea, although no differences reached statistical significance.

The mean central subfield thickness for all eyes was $276 \mu \mathrm{m}$, compared with $270 \mu \mathrm{m}$ for Heidelberg devicespecific published normative data for patients over the age of 60 years. ${ }^{11}$ Significant differences $(P>0.05)$ in macular thickness were detected in three of nine subfields between PD patients and published normal values. In PD subjects, the outer superior subfield was $2.8 \%$ thinner $(P=0.026)$, while the outer nasal and inner inferior subfields were $2.8 \%$ $(P=0.016)$ and $2.7 \%(P=0.001)$ thicker than published normal values, respectively (Table 3 ). No significant differences were detected in the other six subfields. Applying a Bonferroni correction, which decreases false positives but increases false negatives, sets the $P$ value at 0.0056 , at which only the inner inferior subfield reaches significance.

Table I Patient demographics

\begin{tabular}{lll}
\hline & PD & Controls \\
\hline Eyes $(\mathrm{n})$ & 18 & 19 \\
Patients $(\mathrm{n})$ & 9 & 16 \\
Females $(\mathrm{n}, \%)$ & $5(56 \%)$ & $10(62 \%)$ \\
Average age & 64 & 67 \\
Age range & $52-75$ & $50-8 \mathrm{I}$ \\
\hline
\end{tabular}

Abbreviation: PD, Parkinson's disease.
Table 2 Mean inner retinal thickness in Parkinson's disease patients versus controls

\begin{tabular}{|c|c|c|c|}
\hline \multirow[t]{2}{*}{ Location } & \multicolumn{2}{|c|}{$\begin{array}{l}\text { Inner retinal thickness } \\
(\mu \mathrm{m})\end{array}$} & \multirow{2}{*}{$\begin{array}{l}P \\
\text { (Mann- } \\
\text { Whitney U) }\end{array}$} \\
\hline & PD & Control & \\
\hline Fovea & $49( \pm 36)$ & $46( \pm 3 I)$ & 0.704 \\
\hline Temporal 0.5 & $107( \pm 15)$ & $107( \pm 20)$ & 0.738 \\
\hline Temporal I.0 & $150( \pm 10)$ & $145( \pm 16)$ & 0.280 \\
\hline Temporal I.5 & $145( \pm \mathrm{II})$ & $|4|( \pm \mid 5)$ & 0.181 \\
\hline Temporal 2.0 & $126( \pm 10)$ & $124( \pm 18)$ & 0.648 \\
\hline Temporal 2.5 & $113( \pm 8)$ & $115( \pm 15)$ & 0.616 \\
\hline Temporal 3.0 & $105( \pm 8)$ & $103( \pm I I)$ & $0.76 \mathrm{I}$ \\
\hline Nasal 0.5 & $122( \pm 18)$ & III ( $( \pm 26)$ & 0.136 \\
\hline Nasal I.0 & $163( \pm 9)$ & $160( \pm 18)$ & 0.171 \\
\hline Nasal I.5 & $158( \pm 9)$ & $164( \pm 20)$ & 0.353 \\
\hline Nasal 2.0 & $149( \pm 14)$ & $149( \pm 19)$ & 1.00 \\
\hline Nasal 2.5 & $144( \pm 16)$ & $139( \pm 19)$ & 0.224 \\
\hline Nasal 3.0 & $155( \pm 19)$ & $146( \pm 19)$ & 0.121 \\
\hline
\end{tabular}

Notes: Mean IRL thickness measurements in $\mu \mathrm{m}$ as measured with the Heidelberg Spectralis ${ }^{\circledast}$ HRA + OCT in patients with PD and age-matched controls. MannWhitney $U$ test used to detect differences $(P \leq 0.05)$.

Abbreviations: PD, Parkinson's disease; IRL, inner retinal layer.

No significant differences were detected in RNFL thickness between PD patients and the Heidelberg normative database values (Table 4). Overall average RNFL thickness was $97 \mu \mathrm{m}$ for PD patients, which matched the normative database value exactly.

\section{Discussion}

To our knowledge, this pilot study is one of the first studies to utilize high-resolution SD-OCT with both automated and

Table 3 Mean macular thickness in Parkinson's disease patients versus published normal values

\begin{tabular}{llll}
\hline Location & \multicolumn{3}{l}{ Mean macular thickness $(\mu \mathrm{m})$} \\
\cline { 2 - 4 } & $\begin{array}{l}\text { PD } \\
(\mathbf{n}=\mathbf{1 8})\end{array}$ & $\begin{array}{l}\text { Normals } \mathbf{a}>\mathbf{6 0} \\
(\mathbf{n}=1 \mathrm{I})\end{array}$ & $\begin{array}{l}\mathbf{P} \\
\text { (Mann- } \\
\text { Whitney U) }\end{array}$ \\
\hline Central subfield & 276 & 263 & 0.150 \\
Inner temporal & 325 & 332 & 0.156 \\
Inner superior & 335 & 329 & 0.322 \\
Inner nasal & 336 & 326 & 0.059 \\
Inner inferior & 334 & 316 & 0.001 \\
Outer temporal & 313 & 322 & 0.105 \\
Outer superior & 321 & 332 & 0.026 \\
Outer nasal & 331 & 320 & 0.016 \\
Outer inferior & 316 & 314 & 0.605 \\
\hline
\end{tabular}

Notes: Mean macular thickness measurements in $\mu \mathrm{m}$ as measured with the Heidelberg Spectralis ${ }^{\circledR}$ HRA+OCT in patients with Parkinson's disease compared with published normal values of subjects over the age of 60. A Mann-Whitney $U$ test was used to detect differences. Significance levels can be set at $P<0.05$ (greater potential for false positives) or $P<0.0056$ using a Bonferroni correction (greater

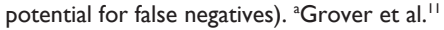

Abbreviation: PD, Parkinson's disease. 
Table 4 Mean retinal nerve fiber layer thickness in Parkinson's disease patients versus normal values

\begin{tabular}{llll}
\hline Location & $\begin{array}{l}\text { Mean RNFL thickness } \\
(\mu \mathrm{m})\end{array}$ & $\begin{array}{l}\boldsymbol{P} \text { (one-sample } \\
\text { t-test) }\end{array}$ \\
\cline { 2 - 3 } & PD & Normals $^{\mathbf{a}}$ & \\
\hline $\mathrm{T}$ & $7 \mathrm{I}$ & 74 & 0.315 \\
$\mathrm{ST}$ & $\mathrm{I} 30$ & 134 & 0.306 \\
$\mathrm{SN}$ & 106 & 102 & 0.550 \\
$\mathrm{~N}$ & 74 & 72 & 0.613 \\
IN & 113 & 105 & 0.363 \\
IT & 139 & 141 & 0.593 \\
Average & 97 & 97 & 0.911 \\
\hline
\end{tabular}

Notes: Mean retinal nerve fiber layer (RNFL) thickness measurements in $\mu \mathrm{m}$ as measured with the Heidelberg Spectralis ${ }^{\circledR}$ HRA+OCT in patients with Parkinson's disease compared with normal values from the Heidelgberg database. A one-sample $t$-test was used to detect differences $(P \leq 0.05)$

Abbreviations: RNFL, retinal nerve fiber layer; T, temporal; ST, superior temporal; $\mathrm{SN}$, superior nasal; N, nasal; IN, inferior nasal; IT, inferior temporal; PD, Parkinson's disease.

manual segmentation to examine retinal changes in PD. ${ }^{7-9}$ Hajee et al demonstrated thinning of the inner ganglion complex (as defined by the specific OCT manufacturer) in PD patients using autosegmentation OCT scans. ${ }^{9}$ Using lower resolution time-domain OCTs, Altinas et al showed reduced macular volume, macular thickness, and RNFL thickness in PD patients, and Inzelberg et al demonstrated significant RNFL thinning in PD patients. ${ }^{7,11}$

Our study failed to show significant reductions in IRL thickness between PD patients and age-matched controls. In fact, our results showed PD patients to have a slightly greater IRL thickness at 11 of 13 points along a section through the fovea, although none of these reached significance. Moreover, no significant differences were found in RNFL thickness between PD patients and normal values.

However, our results did show significant differences in macular thickness at three of nine subfields, including the outer superior, outer inferior, and outer nasal subfields. One of the three subfields showed decreased macular thickness in the PD patients compared to published normal values, while two showed significant increases in macular thickness. This did not agree with the findings of Altinas et al, which showed thinning of the outer inferior, outer nasal, outer temporal, and inner superior subfields, and found no significant difference in the outer superior subfield. ${ }^{8}$

Overall, as a small pilot study, this study is limited by the small number of eyes included. However, our sample size of nine patients does not differ greatly from previously published studies (10 patients for Inzelberg et al, 17 patients for Altinas et al, and 24 patients for Hajee et al). A larger cohort of patients, followed prospectively, and examined with high-resolution SD-OCT, is necessary to clarify the precise relationship between retinal structural changes and PD.

A strength of the current analysis lies in its use of SD-OCT as opposed to time-domain OCT. The increased resolution of SD-OCT allows for more precise and accurate measurements of retinal layer thickness. SD-OCT also offers other advantages, ie, true volumetric calculations due to significantly less interpolation, image registration enabling accurate and reproducible long-term monitoring, and improved image segmentation facilitating analysis of specific retinal layers. Moreover, our study, in addition to the automated segmentation, utilized manual segmentation, enabling more accurate measurements of specific retinal layers (especially the IRL for which there is currently no automated segmentation). Although automated segmentation with SD-OCT is considerably more reliable than TD-OCT, even with high-resolution $\mathrm{OCT}$, the not infrequent occurrence of segmentation errors is a confounding factor in OCT analysis of PD patients. ${ }^{14}$ Lastly, unlike previous studies, for the macular thickness and RNFL comparisons, we evaluated the measurements in PD in relation to that of the large, validated, and normalized control database provided by the manufacturer (and used routinely for such analysis in glaucoma patients). Previous studies in PD patients used considerably smaller populations of "control" patients typically recruited from other patients who presented to the ophthalmologist.

Overall, our findings do not suggest that IRL thickness or RNFL thickness is reduced at a detectable level with the Heidelberg Spectralis SD-OCT in PD patients. Our results do suggest, however, that changes in overall macular volume may be detectable at specific subfields, which suggests that SD-OCT could potentially be correlated with disease severity and used as a biomarker for PD progression. However, as with all previous OCT studies in PD patients, this study involved a small cohort of patients, and these and other results should be viewed as preliminary findings. Larger, longitudinal, prospective studies are needed to explore further the potential relationship between retinal findings and OCT measurements. Until such studies are undertaken, the routine clinical use of OCT for evaluation of PD patients must be taken with great caution.

\section{Acknowledgments}

This project was funded, in part, by the American Parkinson Disease Association Medical Student Fellowship Program and Research to Prevent Blindness. Claire Henchcliffe is supported in part by the Daisy and Paul Soros Clinical Scholarship in Neurology. 


\section{Disclosure}

The authors have no proprietary or financial interest in any aspect of this report.

\section{References}

1. Parkinson J. An Essay on the Shaking Palsy. London: Sherwood, Neely, and Jones; 1817.

2. Frederick JM, Rayborn ME, Laties AM, Lam DM, Hollyfield JG. Dopaminergic neurons in the human retina. J Comp Neurol. 1982;210: 65-79.

3. Harnois C, di Paola T. Decreased dopamine in the retinas of patients with Parkinson's disease. Invest Ophthalmol Vis Sci. 1990;30:2473-2475.

4. Bodis-Wollner I. Visual deficits related to dopamine deficiency in experimental animals and Parkinson's disease patients. Trends Neurosci. 1990;13:296-302.

5. Price MJ, Feldmen RG, Adelberg D, Kayne H. Abnormalities in color vision and contrast sensitivity in Parkinson's disease. Neurology. 1992; 42:887-890.
6. Djamgoz MB, Hankins MW, Hirano J, Archer SN. Neurobiology of retina dopamine in relation to degenerative states of the tissue. Vision Res. 1997;37:3509-3529.

7. Inzelberg R, Ramirez JA, Nisipeanu P, Ophir A. Retinal nerve fiber layer thinning in Parkinson's disease. Vision Res. 2004;44:2793-2797.

8. Altintas O, Iseri P, Ozkan B, Caglar Y. Correlation between retinal morphological and functional findings and clinical severity in Parkinson's disease. Doc Ophthalmol. 2008;116:137-146.

9. Hajee ME, March WF, Lazzaro DR, et al. Inner retinal layer thinning in Parkinson's disease. Arch Ophthalmol. 2009;127:737-741.

10. Bodis-Wollner I. Retinopathy in Parkinson disease. J Neural Transm. 2009;116:1493-1501.

11. Grover S, Murthy RK, Brar VS, Chalam KV. Normative data for macular thickness by high-definition spectral-domain optical coherence tomography (Spectralis). Am J Ophthalmol. 2009;148:266-271.

12. Hoehn M, Yahr M. Parkinsonism: Onset, progression and mortality. Neurology. 1967; 17:427-442.

13. Giani A, Cigada M, Esmaili DD, et al. Artifacts in automatic retinal segmentation using different optical coherence tomography instruments. Retina. 2010;30:607-616.
Clinical Ophthalmology

\section{Publish your work in this journal}

Clinical Ophthalmology is an international, peer-reviewed journal covering all subspecialties within ophthalmology. Key topics include: Optometry; Visual science; Pharmacology and drug therapy in eye diseases; Basic Sciences; Primary and Secondary eye care; Patient Safety and Quality of Care Improvements. This journal is indexed on

\section{Dovepress}

PubMed Central and CAS, and is the official journal of The Society of Clinical Ophthalmology (SCO). The manuscript management system is completely online and includes a very quick and fair peer-review system, which is all easy to use. Visit http://www.dovepress.com/ testimonials.php to read real quotes from published authors. 